\title{
RAPID LOCAL AREA MODELING FOR CONSTRUCTION AUTOMATION
}

\author{
Yong-Kwon Cho \\ Corresponding author, Assistant Professor, University of Wisconsin at Platteville \\ Email: choyo@uwplatt.edu \\ Carl T. Haas \\ Associate Professor, University of Texas at Austin \\ Kamel S. Saidi \\ Graduate Research Assistant, University of Texas at Austin \\ Katherine A. Liapi \\ Assistant Professor, University of Texas at Austin \\ S.V. Sreenivasan \\ Associate Professor, University of Texas at Austin
}

\begin{abstract}
Construction equipment is rapidly being retrofitted with robotic capabilities for improved control and safety. To operate this equipment effectively, an operator must constantly relocate the equipment and position objects within the work environment. Current commercially available equipment technology does not provide real-time information to the operator and requires costly and computationally intensive computer hardware and software. A man-machine balanced rapid local area modeling technique is developed in this research and implemented on a large scale hydraulic manipulator. Through experimentation, this study showed that the man-machine balanced technique can significantly reduce modeling time and accuracy and therefore improve the control and operation of automated equipment.
\end{abstract}

Keywords: construction automation, man-machine interface, graphical simulation, manipulator, $\mathrm{CAD}$, laser rangefinder

\section{INTRODUCTION}

Improving the operating efficiency and safety of hydraulically-actuated construction equipment has become one of the leading motivators behind construction automation efforts. Another motivation for automating construction equipment using robotic controls has been to remove the human operator from the working environment. Reducing or eliminating a worker's exposure to the risks associated with construction equipment operation sets the stage for robotic manipulators to deliver their potential benefits [2].

Controlling equipment in an unstructured workspace can be very difficult and dangerous due to the operator's inability to gather continuous visual feedback from the changing work environment. Since construction sites are characterized by extreme variations in lighting, temperature, humidity, sound, and air quality, they are somewhat unpredictable.
Loud noise emanating from equipment may contribute in a large part to miscommunication between the operator and a guide on whom the operator relies for geometric information about obstructed areas. An operator's limited vision may also cause fatal collisions between the end-effector (e.g., a man-lift basket or a concrete pump boom) and nearby structures or people working in the vicinity.

Visualizing geometric information using highlydescriptive 3D models can significantly improve equipment control in several construction automation applications such as heavy lifting, earth moving, material handling, and infrastructure repair and maintenance [4]. Optical procedures for measuring the geometric shapes of objects in a work environment play an important role in state-of-the-art process automation and quality control systems [1]. Since these procedures also have a number of advantages, considerable effort has been devoted to the development of applications for improved 
visualization of remote and obscured areas for equipment interaction with various environments.

This paper presents a new method for rapid modeling and visualization of a local area based on geometric information about objects obtained using simple sensors (such as a single-axis laser rangefinder and a video camera) for better planning and control of construction equipment operations in unstructured workspaces.

\section{RAPID WORKSPACE MODELING}

\subsection{Man-Machine Balanced Local Area Sensing}

By incorporating human perception, the graphical modeling approach outlined here has the potential to reduce data acquisition time and the need for computationally intensive and expensive image processing.

Since most target objects are known and manmade, they can be graphically generated and stored with complete properties in a computer database. In a design process, pre-stored models include all global properties of the real-world objects such as their shape, texture, color, and roughness. A library of prestored models (related to design elements), with manual guidance, can provide graphic representations of forms that can be matched and fitted to sensed data from 3D position sensors deployed in the work environment

(Figure

$1)$.

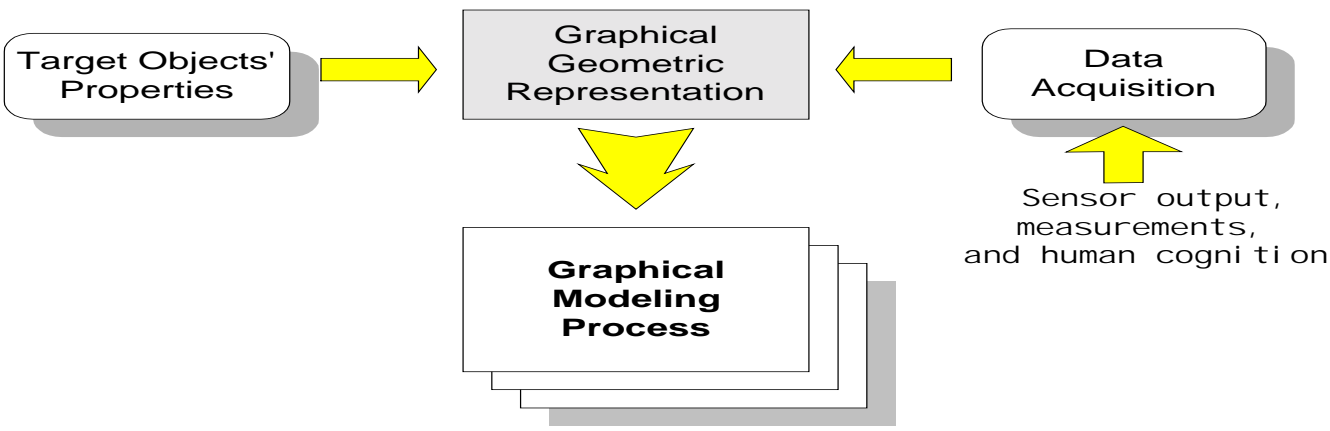

Figure 1. Model Structure of the Human-Assisted Graphical Modeling Process

Successful automation requires a balance between exploitation of a machine's ability to efficiently process a vast amount of information while also executing tasks with high accuracy and force, and a human's ability to react intelligently to unforeseen circumstances or to extract patterns out of apparent chaos [5]. By strategically incorporating human assistance, geometric data acquisition of realworld objects can be simplified and accelerated considerably since distance and orientation may be acquired without the need for area range maps. Furthermore, rapid object recognition using human logic can be achieved without computationally complex and intensive image analysis.

For construction applications, the speed of modeling and the precise measurement of the distances between equipment and target objects in a changing environment are critical issues. In contrast to the work environments of other industries such as manufacturing and architectural design, construction has a more dynamic environment, which allows little time to gain precise geometric information. The University of Texas at Austin has been conducting research to improve the control of a computercontrolled large scale manipulator (LSM). While there have been several improvements in the hydraulically-actuated LSM's control, it still encounters problems when interacting with other objects in its environment. Depth perception, interference detection, and optimized path planning are some examples of these unresolved problems. To resolve these issues, the current research focuses on providing rapid 3D workspace information for operating the LSM.

\subsection{Geometric Data Acquisition for 3D Object Modeling}

Ideally, the position and orientation of most solid objects can be determined using only the Cartesian coordinates of a certain number of vertices, or points on the edges or faces of an object. The number of vertices or points on the edge or a surface depends on the geometric features of the solid object. Two different types of solid modeling methods -parametric modeling and complex modeling- were implemented.

Parametric modeling defines the size and geometry of features and creates relationships between them. The geometric descriptions of parametric models composed of primitives (simple shapes such as a box, sphere, cylinder, or cone), which could reflect construction materials such as pipes or box-beams, were examined to obtain the minimum required points with regard to their position 
and orientation required to define them.

Unlike parametric models, most complex models (such as equipment, facility, or structure) need to be pre-designed and imported into a CAD environment using a fitting and matching process based on the measured target object's geometric data. Hence, in theory, even with only a partial view of an object, or even if the object has occluded areas, as long as a few points on the surface can be measured, and as long as the partially-visible object can be recognized by a human operator, the proposed method can accurately represent the object.

The aforementioned modeling methods produce a very precise graphical model of the immediate environment of the construction equipment and significantly reduce sensor data-acquisition time and computer processing, when compared to other laser range scanning 3D modeling methods. These other methods require substantial time for merging and interpreting dense clouds of scanned laser range data. It is likely that an ideal approach will use the method described here, but relaxed either to tolerate some error or to allow sparse point acquisition and subsequent interpretation.

The LSM control system calculates a series of joint angles through which to move the joints in order for the end-effector frame to move from its initial location (frame $\{E\}$ ) in a smooth manner to its final location (near frame $\{G\}$ ) (See Figure 2). Then, the link transformations are multiplied together to find the single transformation that relates frame $\{G\}$ to frame $\{B\}$. As with vectors and rotation matrices, a symbol $\mathrm{T}$ is called a transformation operator [3]. Here, ${ }_{G}^{B} T$ describes frame $\{\mathrm{G}\}$ relative to frame $\{\mathrm{B}\}$ and forms the following transform equation:

$$
{ }_{G}^{D} T={ }_{C}^{D} T{ }_{E}^{C_{E}} T{ }_{G}^{\mathrm{c}} T
$$

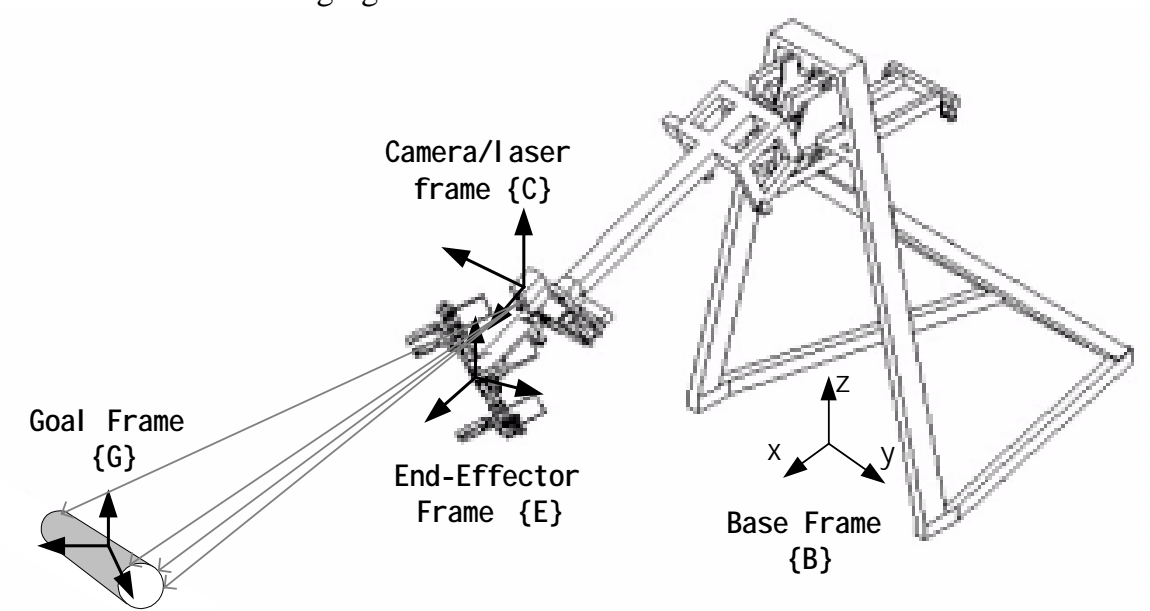

Figure 2. Geometric Parameter Measurement by a Laser on the LSM

In a demonstration to illustrate the approach being developed, a pipe was placed in front of the LSM, and three points on the edge of one end and a point on the edge of the other end were measured by the laser system mounted on the LSM. A solid parametric model was generated in a CAD environment based on the measured points relative to the base coordinate system (Figure 3). The three points on the left edge of the pipe define the plane and the diameter of the circular base while the fourth point defines the length of the pipe.
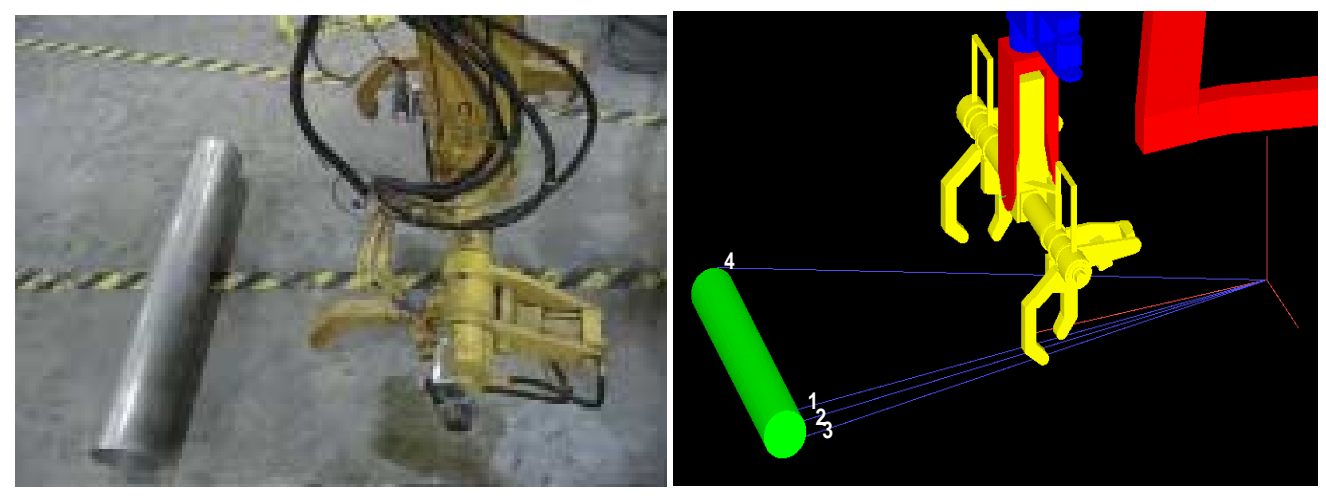

Figure 3. The Fitting and Matching Process for the Pipe 


\section{SYSTEM DESCRIPTION}

\subsection{The Large Scale Manipulator (UT)}

The Large Scale Manipulator (LSM) at the University of Texas at Austin is a 6 Degree of Freedom (DOF) hydraulically-actuated manipulator that was originally designed for handling pipes in construction operations. In its conventional configuration, it is attached to the boom of a rough terrain mobile crane. To allow research to continue in a more controlled environment, the LSM was moved to an indoor facility at the Field Systems and Construction Automation Laboratory.

In addition to manual control, autonomous control is currently used to operate the LSM. Motion commands are preprogrammed into a computer and sent to the LSM to achieve the desired motion. The computer controls the LSM using sensors that determine joint positions and actuates each joint until the desired position is reached within specified tolerances.

\subsection{The Laser/Camera-Based Data Acquisition System}

In order to manually acquire position data of materials, facilities, and structures in the manipulator's local work environment, a laser rangefinder, a camera, and a tele-operated pan-andtilt unit (PTU) were installed on the LSM (Figure 4). Once a target's position is measured by the camera/laser system, the spatial relationship between the target and the end-effector of the manipulator can be kinematically determined. For this study, a singleaxis laser rangefinder was used.

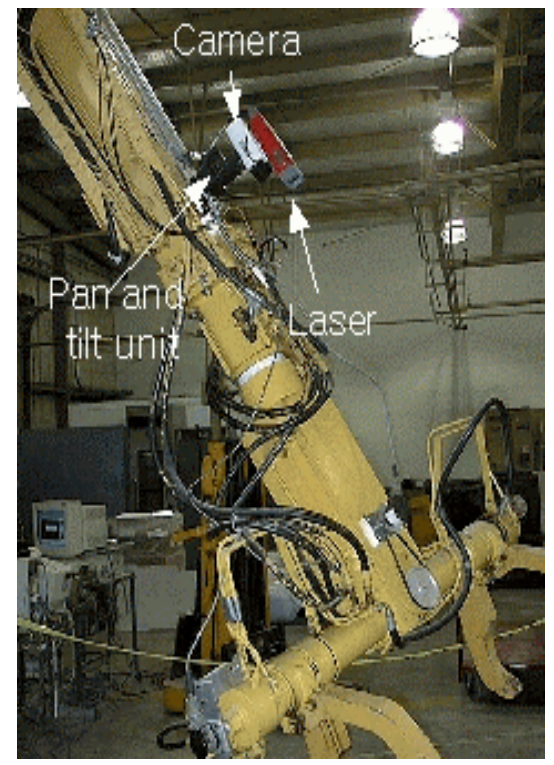

Figure 4. The Laser and Camera System on the LSM

\subsection{The Kinematics}

As illustrated in Figure 5, the LSM has a swing joint, a lift joint, a telescopic joint and a hand that has a 3 DOF wrist joint. The camera/laser rangefinder is mounted on the LSM at point A. The camera/laser rangefinder can be moved using the swing, lift and telescopic joints. It also has an in-built pan and tilt joints. The location of the laser spot is therefore affected by the swing angle, lift angle, telescopic length, pan angle, tilt angle, and the length of the laser beam. Therefore, the 6 DOF forward kinematics for the camera/laser system mounted on the LSM can be used to solve for the position of the laser's target spot. Forward and inverse kinematics calculations are required to relate the camera/laser sensed coordinates to the manipulator coordinates.

The camera/laser coordinate system provides the final target point of the laser beam with respect to the LSM's base coordinate system using forward kinematics. The required joint angles of the LSM are then calculated using inverse kinematics and the endeffector moves to the desired location.

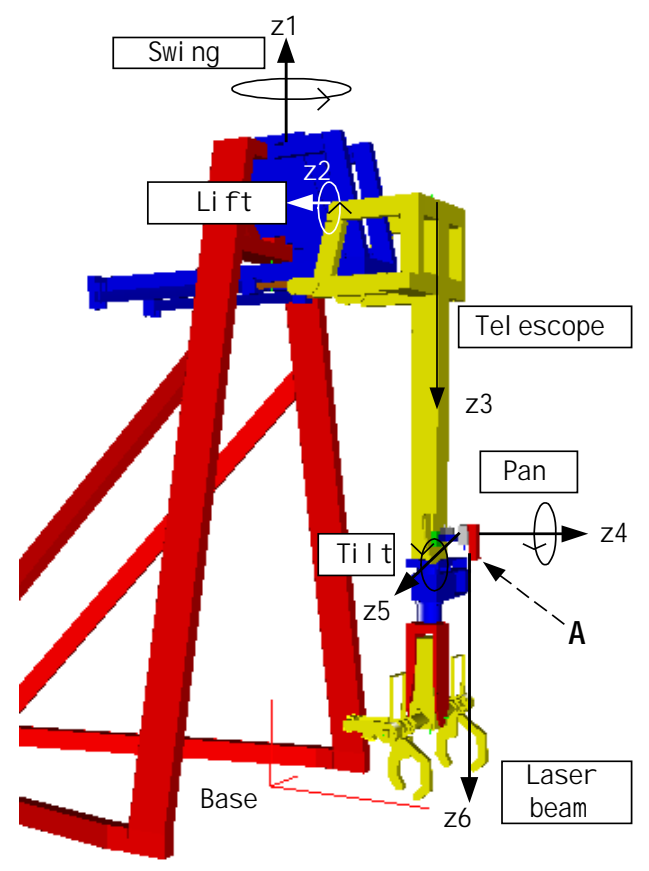

Figure 5. Six DOF Kinematic Configuration for the Laser/LSM System

\subsection{The Overall System Architecture}

Figure 6 illustrates the overall system architecture described in this section. 


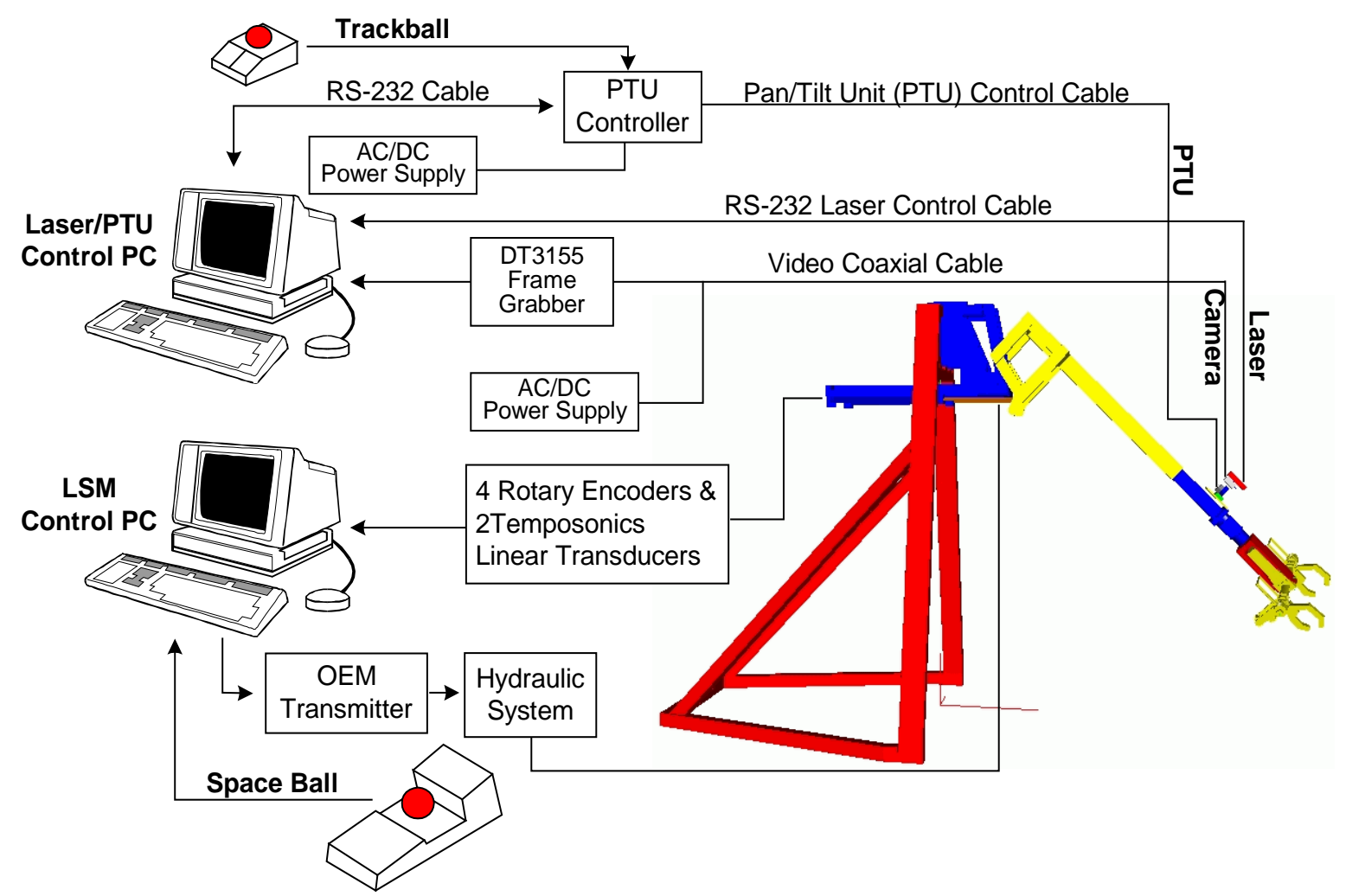

Figure 6. The Overall System Architecture

\section{PERFORMANCE TESTS}

\subsection{Off-line Programming}

Graphical off-line programming enables users to examine a robot's movement through graphical simulation and to find errors in the programming that could lead to fatal accidents while the robot undergoes motion. The general benefits of off-line programming for this study include:

- Interference detection,

- Manipulator reach and range of motion study,

- Final design simulation,

- Cycle time estimations, and

- Better understanding of cyclic processes.

Based on the spatial data (in the 3rd column of Table 1) obtained from the graphical simulation, real task execution was conducted on the LSM's test bed. The tests included the horizontal placement of a wooden beam and an aluminum pipe and the vertical placement of aluminum pipes on a pipe rack (Table 1). By giving a motion script derived from the graphical simulation to the LSM control system, the LSM automatically moves to the inputted goal position.

The main purpose of the graphical simulation and real-time execution tests was to verify the overall proposed method including the usefulness of the hardware and software platforms developed in this study. The tests proved that the overall system effectively improved the operation of the LSM. The most crucial issue in the off-line programming test was the correspondence between the two systems, the graphic workspace and the real world workspace. The test results indicated that, within the limited tasks performed in the experiments, the overall correspondence accuracy was acceptable for the LSM's operation.

Regardless of the complexity and size of the objects, the average processing time for graphically modeling an object was approximately 2 minutes using the current prototype system. Thus, it is likely that this graphical modeling approach would be more effective for modeling large and complex objects than small and simple ones.

In order to increase the modeling speed, as an on-going project, this research is currently focusing on improving the data acquisition and modeling process by optimizing the manual guidance of sensor data acquisition and minimizing human interruption in the graphical modeling process. 
Table 1. Graphical Simulation and Real Task Execution

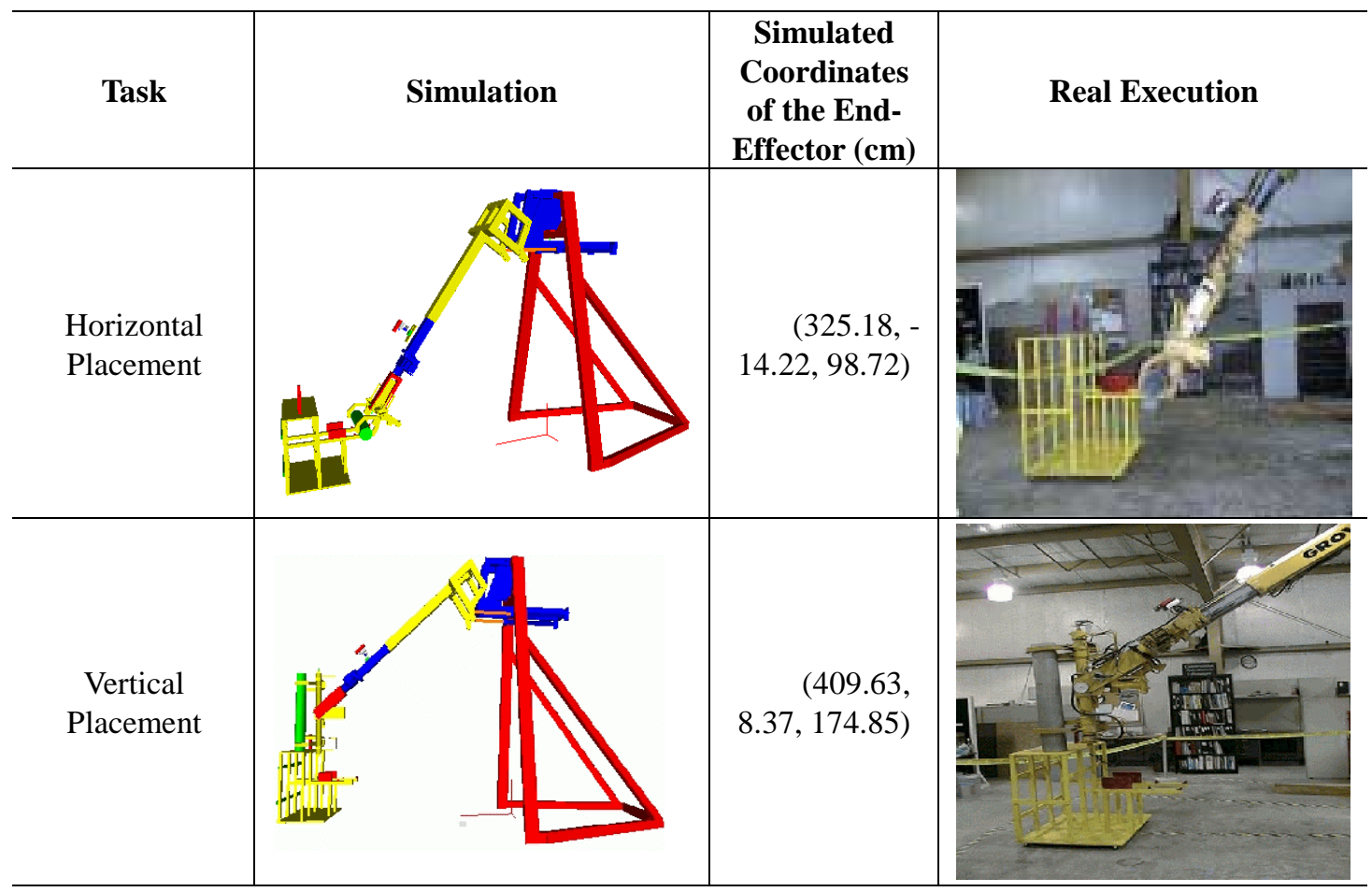

Current research is also focusing on: (1) fitting objects to sparse range point clouds, and (2) developing methods to efficiently build virtual boxes around construction environment elements for realtime obstacle avoidance for the manipulator.

\section{CONCLUSION}

In preliminary work, a new method for rapidly modeling and visualizing geometric information in construction environments has been developed by combining human perception with highly descriptive CAD models using simple sensors. The method was shown to significantly reduce modeling time while potentially increasing modeling accuracy. The test results in this paper indicate that potential real-time $3 \mathrm{D}$ modeling and visualization of the workspace is achievable with very simple and low-cost sensor fusion techniques. The potential impact of this research includes safer and more efficient operation of computer-assisted construction and maintenance equipment.

\section{Acknowledgements}

This paper is based on research funded by the National Science Foundation (Grant \#: CMS0000137). The authors gratefully acknowledge their financial support and encouragement throughout this study.

\section{REFERENCES}

[1] Albrecht, P. and Michaelis, B. "Improvement of the Spatial Resolution of an Optical 3-D Measurement Procedure", IEEE Transactions on Instrumentation and Measurement, 47(1), 1998.

[2] Bernold, L. and Huang X. "CAD-Integrated RealTime Control for Robotic Excavation and PipeLaying: Development and Field Testing", Final Report, Federal Highway Administration, 1996.

[3] Craig, J. Introduction to Robotics: Mechanics \& Control, Addison-Wesley, 1986.

[4] Haas, C., Skibniewski, M., and Budny, E. "Robotics in Civil Engineering, Microcomputers in Civil Engineering”, 371-381, October, 1995.

[5] Kim, Y.S. and Haas, C. “A Model for Automation of Infrastructure Maintenance using Representational Forms", Journal of Automation in Construction, 10(1), 2000. 